\title{
A SCALING RELATION WITH ROLLING TACHYON FIELDS AND COSMIC ACCELERATION
}

\author{
Indrajit Mondal \\ Department of Physics, \\ Ramakrishna Mission Vivekananda Educational and Research Institute, \\ Belur Math, Howrah-711202, India \\ Email: indrajit.mondal@rkmvu.ac.in
}

\begin{abstract}
This theoretical investigation focuses on a scaling relation for rolling tachyon fields $\mathrm{T}(\mathrm{t})$ for a homogeneous cosmology with the Friedmann-Lemaitre-Robertson-Walker (FLRW) metric. A Lagrangian is set up and the Euler-Lagrangian equations are solved for it. The tachyon fields obtain from EulerLagrangian equations exhibits late-time acceleration i.e., cosmic acceleration with negative pressure.
\end{abstract}

Keywords: rolling tachyon, Lagrangian, Hubble parameter, cosmic acceleration, FLRW metric, homogeneous cosmology, early Universe

\section{INTRODUCTION}

In recent years the expansion of the very early universe has a lot of evidence from WMAP and Planck observations of the Cosmic Microwave Background. It suggests that the early universe underwent a period, of inflation when its expansion rate was accelerating (Guth 1981). The study of inflation described that the inflationary phase is driven by the potential energy of the canonical scalar field[1, 2, 3, 4, 5, 6, 7, 8, 9, 10, 11, 12] (inflaton) whose dynamics is described by the standard Lagrangians and the corresponding Klein-Gordon equation. There are different models of scalar fields to describe inflation. Tachyon field[1, 2, 3, 4] is one of the important candidates among them.

The Lagrangian density of the tachyon field which is described by effective field theory in flat space is $[1,2,3,4]$

$$
L=-V(T) \sqrt{1+\eta^{\mu \nu} \partial_{\mu} T \partial_{\nu} T}-----(1)
$$


Where

$T$ : tachyon field

$V(T)$ : tachyon potential

$\eta_{\mu \nu}=\operatorname{diag}(-,+,+,+$,$) : metric of Minkowski spacetime.$

The tachyon potential $V(T)$ has a positive maxima at the origin and has a minimum at $T=T_{0}$, where the potential vanishes, $T_{0}$ is taken to lie at infinity.[1]

In Minkowski space-time, the rolling down towards its minimum value is described by a spatially homogeneous but time-dependent solution obtain from the Lagrangian density (from equation (1)) [3, 4]

$$
L=-V(T) \sqrt{1-\dot{T}^{2}}-----(2)
$$

Here the energy density $[1,2,3,4]$

$$
\rho=\frac{V(T)}{\sqrt{1-T^{2}}}-----(3)
$$

and pressure $[1,2,3,4]$

$$
P=-V(T) \sqrt{1-T^{2}}-----(4)
$$

Also, the energy-momentum conservation equation for the FLRW universe is

$$
\dot{\rho}+3 H(\rho+P)=0-----(5)
$$

where $H$ is the Hubble parameter defined in terms of scale factor $a(t)$ as $H=\frac{\dot{a}}{a}$

Now using the values of $\rho$ and $P$ from equation (3) and (4) in equation (5), we get

$$
\begin{aligned}
& \frac{d}{d t}\left[\frac{V(T)}{\sqrt{1-T^{2}}}\right]+3 H\left[\frac{V(T)}{\sqrt{1-T^{2}}}-V(T) \sqrt{1-T^{2}}\right]-----(6) \\
& \Rightarrow \frac{\frac{d}{d T} V(T)}{V(T)} \frac{1}{\dot{T}}+\frac{\ddot{T}}{\dot{T}\left(1-T^{2}\right)}+3 \frac{\dot{a}}{a}=0-----(7)
\end{aligned}
$$

Now taking $V(T)=$ constant, we get from the above equation

$$
\frac{\ddot{T}}{\grave{T}\left(1-T^{2}\right)}+3 \frac{\dot{a}}{a}=0-----(8)
$$

Hence it will be 


$$
\frac{d}{d t}\left[\ln \frac{\dot{T}}{\sqrt{\left(1-T^{2}\right)}}\right]+3 \frac{d}{d t}[\ln a]=0------(9)
$$

Now integrating the above equation one gets the scaling relation for the Tachyon field

$$
\frac{\dot{T}}{\sqrt{\left(1-\dot{T}^{2}\right)}}=C a^{-3}-----(10)
$$

where $C$ is constant.

\section{Lagrangian of rolling tachyon:}

Now using the above scaling relation and the zero-zero component of Einstein's field equations an expression for the Lagrangian for the rolling tachyon field can be obtained for the FLRW metric.

Here we work with the FLRW metric

$$
d S^{2}=-d t^{2}+a^{2}(t)\left[\frac{d r^{2}}{\left(1-k r^{2}\right)}+r^{2}\left(d \theta^{2}+\sin ^{2} \theta d \phi^{2}\right)\right]------(11)
$$

and the zero-zero component of Einstein's field equation

$$
R_{00}-\frac{1}{2} g_{00} R=k T_{00}------(12
$$

From equation (11) and equation (12), one gets

$$
\frac{k}{a^{2}}+H^{2}=\frac{8 \pi G}{3} \rho-----(13
$$

Now taking curvature constant $k=0$, we get

$$
\rho=\frac{3 H^{2}}{8 \pi G}-----(14
$$

Using the value of $\rho$ from equation (3)

$$
\frac{V(T)}{\sqrt{1-T^{2}}}=\frac{3 H^{2}}{8 \pi G}-----(15)
$$

Now, the Lagrangian density

$$
L=-V(T) \sqrt{1-\dot{T}^{2}}=--V(T)\left[\frac{1}{\sqrt{1-\dot{T}^{2}}}-\frac{\ddot{T}^{2}}{\sqrt{1-\dot{T}^{2}}}\right]------(16)
$$


Using equation (10) and equation (15) in equation (16), one gets

$$
L=\frac{-3 H^{2}}{8 \pi G}+2 C a^{-3} \dot{T} V(T)-----(17)
$$

Let $C_{1}=\frac{3}{8 \pi G}$ and $q=\ln a$

Therefore,

$$
L=-C_{1} \dot{q}^{2}+2 C V(T) \dot{T} e^{-3 q}-----(18
$$

This new lagrangian has two generalised co-ordinates $\mathrm{q}(\mathrm{t})$ and $\mathrm{T}(\mathrm{t})$.

Now using the scaling equation from equation (10), one gets

$$
\begin{gathered}
\dot{T}(t)=\left(\frac{C^{2}}{a^{6}+C^{2}}\right)^{\frac{1}{2}}-----(19) \\
T(t)=\int\left(\frac{C^{2}}{a^{6}+C^{2}}\right)^{\frac{1}{2}} d t+C_{2}-----(20)
\end{gathered}
$$

where $C_{2}$ is an integral constant.

For a very small value of $C$, the above equations will be

$$
\begin{gathered}
\dot{T}(t)=\left(\frac{C}{a^{3}(t)}\right)-----(21) \\
T(t)=C \int a^{-3}(t) d t+C_{2}------(22)
\end{gathered}
$$

\section{Deceleration parameter $Q$ :}

Using equation (21), one gets

Deceleration parameter $Q=\frac{-a(t) \ddot{a}(t)}{a^{2}(t)}=-4+3 \frac{\dot{T}(t) \dddot{T}(t)}{T^{2}(t)}------(23)$

So, from the above equation $Q \leqslant-1$, i.e., the universe will be accelerated when

$$
\frac{\dot{T}(t) \dddot{T}(t)}{\ddot{T}^{2}(t)} \leqslant 1------(24)
$$

\section{Equation of state parameter $\omega$ :}

Equation of state parameter is given by 


$$
\omega=\frac{P}{\rho}-------(25)
$$

Hence from equation (3) and (4), we get

$$
\omega=\frac{P}{\rho}=-\left(1-\dot{T}^{2}\right)------(25)
$$

Therefore $-1 \leqslant \omega \leqslant 0$

This satisfies the dark energy pressure condition which is negative i.e., negative pressure generates late-time cosmic acceleration.

\section{CONCLUSION}

The importance of this work is that Einstein's gravity and certain rolling tachyon field theories, coupled to the Lagrangian, can lead to similar cosmological scenarios when a certain scaling relation involving the tachyon fields. In this work, we first discuss a rolling tachyon with Lagrangian density in flat space $L=-V(T) \sqrt{1-T^{2}}$. The modified Friedman equations are subjected to the constraint $\frac{\dot{T}(t)}{\sqrt{\left(1-T^{2}(t)\right)}}=C a(t)^{-3}$ and solutions obtained for the scale factor $a(t)$. The tachyon field $\mathrm{T}(\mathrm{t})$, deceleration parameter $\mathrm{Q}$, and equation of state parameter are then determined. The results we have obtained and presented here confirm that the rolling tachyon field is an important model to explain the inflation of the very early universe.

\section{REFERENCES:}

1. Sen, A.: Tachyon Matter. JHEP07 (2002)

2. Sen, A.: Rolling Tachyon. JHEP04 (2002)

3. Sen, A.: Fundamental Strings in open string theory at the tachyonic vacuum. J Math Phys 42, 2844 (2001)

4. Gibbons, G. W.: Cosmological Evolution of the Rolling Tachyon. Phys. Lett. B 537 (2002)

5. Chimento, L.P.: Extended tachyon field, Chaplygin gas, and solvable k- essence cosmologies.Phys.Rev.D, 69, 123517 (2004)

6. Scherrer, R.J.: Purely kinetic k-essence as Unified Dark Matter. Phys.Rev.Lett, 93, 011301 (2004)

7. Gangopadhyay, D., Mukherjee, S.: Logarithm of the scale factor as a generalised co-ordinate in a Lagrangian for dark matter and dark energy. Phys.Lett.B, 665, 121 (2008)

8. Gangopadhyay, D., Mukherjee, S.: F (R) Gravity with K-essence scaling relation and cosmic acceleration. Journal of Mathematical Sciences \& Computational Mathematics, Vol. 1, No. 4, (2020) 
9. Malaquarti, M., Copeland, E.J., Liddle, A.R, Trodden, M.: A new view of k-essence. Phys.Rev.D, 67, $123503(2003)$

10. Copeland, E.J., Sami, M., Tsujikawa, S.: Dynamics of dark energy. Int.Jour.Mod.Phys.D, 15, 1753 (2006)

11.Starobinsky, A. A.: A New Type of Isotropic Cosmological Models Without Singularity. Phys. Lett.B, 91, 99 (1980)

12. Linde, A.: Chaotic inflation. Phys. Lett.B, 129, 177 (1983) 